\title{
La Bibliothèque du Parlement québécois, son lectorat et les services
}

\section{Quebec's Bibliothèque du Parlement: its audience and services La biblioteca parlamentaria de Québec, su clientela y sus servicios}

\section{Gaston Bernier et Philippe Sauvageau}

Volume 54, numéro 2, avril-juin 2008

Topographie du Québec documentaire

URI : https://id.erudit.org/iderudit/1029315ar

DOI : https://doi.org/10.7202/1029315ar

Aller au sommaire du numéro

Éditeur(s)

Association pour l'avancement des sciences et des techniques de la documentation (ASTED)

ISSN

0315-2340 (imprimé)

2291-8949 (numérique)

Découvrir la revue

Citer cet article

Bernier, G. \& Sauvageau, P. (2008). La Bibliothèque du Parlement québécois, son lectorat et les services. Documentation et bibliothèques, 54(2), 87-90.

https://doi.org/10.7202/1029315ar
Résumé de l'article

La bibliothèque des parlementaires québécois, aujourd'hui Bibliothèque de l'Assemblée nationale, a toujours joué le rôle de bras documentaire des législateurs. Collections et services sont taillés pour s'ajuster aux besoins de ces derniers. Cependant, depuis toujours peut-on dire, on y accueille les fonctionnaires de l'État, les citoyens, les chercheurs ou les étudiants qui veulent consulter les collections conservées sur place et dont l'organisation et les instruments de recherche leur simplifient le travail.
Tous droits réservés (c) Association pour l'avancement des sciences et des techniques de la documentation (ASTED) et Corporation des bibliothécaires professionnels du Québec (CBPQ), 2008
Ce document est protégé par la loi sur le droit d'auteur. L'utilisation des services d’Érudit (y compris la reproduction) est assujettie à sa politique d'utilisation que vous pouvez consulter en ligne. 


\title{
La Bibliothèque du Parlement québécois, son lectorat et les services
}

\author{
GASTON BERNIER \\ Bibliothécaire retraité \\ Directeur de la Bibliothèque de l'Assemblée nationale de 1994 à 2000 \\ gastonbernier@assnat.qc.ca
}

\section{PHILIPPE SAUVAgEAU}

Directeur de la Bibliothèque de l'Ássemblée nationale du Québec

psauvageau@assnat.qc.ca

\section{RÉSUMÉ | ABSTRACTS| RESUMEN}

La bibliothèque des parlementaires québécois, aujourd'hui Bibliothèque de l'Assemblée nationale, a toujours jouéle rôle de bras documentaire des législateurs. Collections et services sont taillés pour s'ajuster aux besoins de ces derniers. Cependant, depuis toujours peut-on dire, on y accueille les fonctionnaires de l'État, les citoyens, les chercheurs ou les étudiants qui veulent consulter les collections conservées sur place et dont l'organisation et les instruments de recherche leur simplifient le travail.

\section{Quebec's Bibliothèque du Parlement : its audience and services}

The bibliothèque des parlementaires québécois, known today as the Bibliothèque de l'Assemblée nationale, has always played an important role in providing legislators with access to information. Library collections and services are designed to answer the specific needs of its constituents. Since its inception, however, the library welcomes public servants, citizens, researchers and students wanting to consult its physical collection, and for whom its organization and research tools facilitate their work.

\section{La biblioteca parlamentaria de Quẹ́bec,} su clientela y sus servicios

La biblioteca de los miembros del parlamento de Québec, mejor conocida bajo el nuevo nombre de Biblioteca de la asamblea nacional, ha sido siempre el instrumento informativo por excelencia de los legisladores. Es en función de las exigencias y las necesidades especificas de éstos últimos que han sido concebidas las colecciones y los servicios. Sin embargo, podemos afirmar que desde siempre también son bienvenidos en la biblioteca los funcionarios del Estado, los ciudadanos, los investigadores y los estudiantes deseosos de consultar las colecciones que se conservan en ella y cuya organización e instrumentos de búsqueda aspiran a simplificar el trabajo de consulta de todos sus usuarios.
$\mathrm{P}$ oser la QUestion Du lectorat d'une bibliothèque parlementaire ne soulève pas de problème, aussi longtemps qu'on en exclut la dimension pragmatique. Des organismes internationaux ont dressé des inventaires des pratiques locales, lesquelles semblent limpides au premier regard ${ }^{1}$. Cependant, dans le quotidien et à travers les ans ou les conjonctures, les zones grises ou les flottements persistent et les principes mis de l'avant ne se traduisent pas toujours par des pratiques uniformes dans le temps.

Les bibliothèques parlementaires ont.une mission centrale : celle d'être l'instrument documentaire des hommes et des femmes politiques d'un pays ou d'un État. Elle est la même depuis plus de deux siècles, qu'on observe le phénomène en Amérique ou en Europe. En parallèle cependant, les autorités politiques confient à l'occasion d'autres mandats à ces bibliothèques : composer une collection nationale et conserver l'ensemble de la production éditoriale du territoire ou portant sur le territoire, regrouper l'ensemble des archives gouvernementales et administratives de l'État, gérer le réseau de bibliothèques publiques, etc. La multiplication des mandats périphériques diversifie les fonctions des bibliothèques législatives, mais le soutien aux parlementaires demeure la mission essentielle, celle qui sert à délimiter les collections à composer, la qualification des employés, les prestations à offrir et les crédits afférents à leur allouer.

La Bibliothèque de l'Assemblée nationale du Québec (B.A.N.Q.); mise sur pied en 1802, a connu bien des situations depuis sa naissance. Les députés ont souhaité ún établissement qui soit à leur service à l'époque, et il l'est demeuré. Pendant cent cinquante ans, ses grandes orientations relevaient d'un comité de parlementaires. Cependant, à la frange, la documentation ${ }^{2}$ réunie sur place a été mise à la disposition des fonctionnaires de l'État, des citoyens, des chercheurs et des étudiants,

1. Association des secrétaires généraux des parlements. 1974: 5-6; Union interparlementaire. 1987 : vol. II, 865; Tanfield, Jennifer. 2000.

2. Laquelle couvre l'ensemble du savoir mais avec une dominante dans les domaines du droit, des sciences politiques, de l'économie et de l'administration publique. Sans oublier les publications gouvernementales et les journaux québécois mais aussi d'autres entités politiques. 
même si les services ont parfois été réglementés. Il en va encore de même aujourd'hui avec la collection numérique en élaboration.

\section{Lectorat parlementaire}

Une bibliothèque parlementaire est une bibliothèque privée ${ }^{3}$ ou, mutatis mutandis, une bibliothèque d'entreprise ou une bibliothèque "spécialisée ». Ses abonnés, avant tout des députés, sont ses patrons ou ses dirigeants. Comme le disait Jean-Charles Bonenfant, «... la Bibliothèque de l'Assemblée nationale doit exister avant tout pour cette assemblée et le personnel technique qui en relève $»^{4}$. En principe, les grandes orientations relèvent de ces derniers selon des formules qui varient dans le temps et dans l'espace.

Le lectorat, quant à lui, comprend également le personnel des secrétariats administratifs, les journalistes parlementaires et diverses agences relevant directement de l'Assemblée, comme c'est le cas au Québec : Protecteur des citoyens, Vérificateur général, Directeur général des élections et commissaire au lobbysme.

L'intégralité des services est mise à la disposition des hommes et des femmes politiques. À leur demande, on rédige des bibliographies, des études, des notes d'intervention ou des rapports de commission, on leur prépare des dossiers de presse et on assure une veille documentaire dans leurs domaines de compétence. En aval, pourrait-on dire, des agents rédigent les comptes rendus des débats antérieurs à 1963, des documentalistes en établissent les tables alphabétiques, indexent les interventions tant des élus contemporains que de ceux du $19^{\mathrm{e}}$ et de la première moitié du $20^{\mathrm{e}}$ siècle, d'autres élaborent les inventaires des dossiers archivistiques confiés à l'Assemblée nationale, des techniciens numérisent des publications parlementaires et des discours ou interventions prononcés à l'extérieur du palais législatif. En somme, les parlementaires/sont la raison d'être de la Bibliothèque et ce qu'elle est correspond, en principe, à leurs besoins, à leurs'desiderata et à leurs centres d'intérêts.

À côté du noyau central formé des parlementaires, on trouve les collaborateurs immédiats de ces derniers et le personnel administratif de l'Assemblée. En gros, les uns et les autres sont à leur service, les uns d'une manière non partisane ou neutre et les autres apportant la nuance ou la coloration politique. Les fonctionnaires parlementaires et les collaborateurs des parlementaires sont actuellement au nombre d'environ 900 ou 1 ooo. À des questionnaires d'associations professionnelles, les dirigeants de la Bibliothèque avaient donné le nombre de $i 114$ et de 1533 pour 1993-1994 et 1994-1995. Tous, qu'il s'agisse des attachés ou des fonctionnaires, sont

3. "Our library if of a private character although a certain number of readers and subscribers are admitted with recommendations from a member of the Legislature" (Lettre datée du 14 octobre 1938 et addressee à Lars Frykholm, bibliothécaire parlementaire de Stockholm; AAN, Fonds Marquis, dossier 2.1).

4. ACBLF. $1969: 10$. habituellement considérés comme les mandataires des élus.

\section{Lecteurs de la Fonction publique}

Le bassin de lecteurs formé par lès employés de l'État - mieux, de l'Administration gouvernementale - est beaucoup plus important numériquement que l'effectif parlementaire au sens large. On y trouve le personnel des ministères et des organismes qui leur sont rattachés et les commissions d'étude ou d'enquête que l'Exécutif met sur pied à l'occasion. On peut même assimiler à ce groupe les bureaux dont il a été question plus haut et qui relèvent de l'Asssemblée nationale.

Les relations de service qui existent entre l'ensemble de ces fonctionnaires - avant tout de la fonction gouvernementale mais aussi de la fonction de contrôle dévolue à l'Assemblée - sont fort différentes de celles qui prévalent dans le milieu parlementaire. Les fonctionnaires de l'État peuvent utiliser sur place la documentation sélectionnée et réunie en fonction des besoins des parlementaires. Il leur faudra faire appel au prêt entre bibliothèques. Au demeurant, les volumes et revues conservés à la B.A.N.Q. sont signalés au catalogue collectif consultable à distance (Cubiq) et, donc, mis à disposition des lecteurs au sens large. Les photocopies leur seront fournies à titre onéreux. Par ailleurs, des prestations, au-delà d'une orientation générale - soumission d'une question de référence, commande d'une bibliographie ou d'une étude - sont hors de leur portée.

Les liens de proximité entre les fonctionnaires du gouvernement et la Bibliothèque se sont distendus au cours des ans. Il fut une époque où, peu nombreux, ils logeaient tous à l'Hôtel du Parlement, qu'on appelait, et pour cause, l'Hôtel du gouvernement, ou dans le voisinage. Dans les années 1960, les ministères ont déménagé et se sont dotés de bibliothèques intérieures. Jusque-là, les ministres ou les chefs de service adressaient des lettres de recommandation au conservateur en faveur de fonctionnaires (les archives conservent des traces de cette pratique). À la même époque, J.C. Bonenfant constatait : « ... depuis quelques années, la bibliothèque... se consacre uniquement au services des membres de l'Assemblée... et des fonctionnaires " (rapport annuel, 15 février 1968). Mais en 1985, l'impact de l'utilisation des bibliothèques de l'Administration, de plus en plus nombreuses et sans doute mieux dotées, et le départ des derniers ministères des bâtiments de la colline parlementaire étaient invoqués lors de la préparation des crédits.

Louverture de la bibliothèque des députés et des conseillers législatifs au personnel de la fonction exécutive na jamais fait l'objet de directives ou de pratiques en bonne et due forme. La nature des institutions parlementaires d'origine britannique, le croisement des fonctions législatives et gouvernementales (les ministres sont des parlementaires) menaient tout naturellement 
à l'utilisation des services documentaires sans qu'il soit nécessaire de le préciser ${ }^{5}$. Au demeurant, la fonction " contrôle » exercée par la Chambre (ou par les Chambres avant 1968) contribuent à la composition de collections adaptées aux préoccupations des parlementaires et, par le fait même, au fonctionnarisme et à l'Éxécutif, et à leurs champs de compétence ou d'intervention.

\section{Les citoyens}

Pour leur part, les citoyens ou les électeurs forment un lectorat extérieur. Si on néglige leur influence quasi souterraine ou géologique, c'est-à-dire à très long terme, sur la composition des collections, on peut dire que les services et les prestations des bibliothèques parlementaires sont dessinés pour la représentation nationale, sans prendre en compte les besoins documentaires immédiats ou latents de la population. Cela ne signifie toutefois pas que ces établissements ne soient pas en mesure de répondre à des préoccupations ou de combler certains besoins documentaires de la population en général.

La bibliothèque de l'Assemblée a été ouverte au public dès 1825 à la suite de la recommandation d'une commission parlementaire ${ }^{6}$. Les conservateurs ont mis en train la pratique, la bonifiant même : ils ont imaginé d'allonger au besoin les heures d'ouverture ${ }^{7}$ afin de favoriser les travailleurs soumis à des horaires fixes, , proposé de mettre sur pied une section scolaire en 1936 (projet qui semble être resté lettre morte), sollicité des calendriers publicitaires d'une entreprise montréalaise ${ }^{8}$ en raison de la présence des citoyens, justifié l'installa-. tion d'un téléphone supplémentaire pour qu'on puisse répondre aux "demandes de renseignements au sujet des concours de la radio $" 9$. En 1955, lors de l'étude des crédits, on exprima le souhait que la Bibliothèque ne soit pas complètement fermée au public pendant la session comme cela était prévu au règlement. En gros, la politique d'ouverture a toujours prévalu. Cependant, la fréquentation des citoyens a diminué dans la seconde moitié du $20^{\mathrm{e}}$ siècle ${ }^{10}$, à la suite de la structuration de la bibliothèque publique de Québec et de la spécialisation progressive des collections composées à l'intention des parlementaires.

5. On retrouve une trace de cette position manifeste dans le Bulletin trimestriel (de la Bibliothèque), vol. $4, n^{\circ} 3$, juillet 1973, p. 24-25: « ... en vertu de la fonction de bibliothèque générale de l'Administration... il incombait à la Bibliothèque... d'assurer le regroupement bibliographique des documents conservés dans les bibliothèques gouvernementales..." ":

6. Gallichan, Gilles.1987 : 6; Journaux de la Chambre d'assemblée...1825 (17 mars) : 361.

7. Proposition de Étienne Parent en 1835 (La Presse, 30 juillet 1921).

8. Lettre datée du 9 janvier 1946 et adressée à Daigle \& Paul (AAN, Fonds Marquis, dossier 1.10).

9. Bordereau daté du 17 janvier 1951 adressé au ministère des Travaux publics (A.A.N., Fonds Marquis, dossier 5.6).

10. J.C. Bonenfant a écrit en 1965 : " A vrai dire, (la bibliothèque) a cessé de servir de bibliothèque publique à la ville de Québec, rôle qu'elle a joué autrefois " (ACBLF, 1965 : 21). Mais, à la même époque, il répondait à un correspondant : « Notre Bibliothèque est ouverte au public..." (Lettre au Frère Gérard Malendant, datée du 26 janvier 1965; Archives de l'Assemblée nationale. Fonds Bonenfant, dossier 1.3).
La fréquentation de la bibliothèque par les citoyens, avant tout de la capitale, était encadrée : le lecteur doit présenter une lettre de recommandation de son député ; il ne peut emprunter plus de deux volumes à la fois durant l'intersession, et il lui faut obligatoirement les rendre avant le début des travaux parlementaires. Il est arrivé que la liste des délinquants ou retardataires soit transmise à l'Orateur (aujourd'hui le président) et que des conservateurs mentionnent - en $1861^{11}$, 1888 et $1889^{12}$ en particulier - des cas de pillage et de désagrément.

La présence du lectorat externe et de son chalandage ont été mesurés pendant certaines périodes. On a précisé que 786 personnes avaient fréquenté la bibliothèque en 1888 et, qu'en 1917, 900 s'étaient inscrites au registre des abonnés. On avait emprunté 17000 en 1890 et Ernest Myrand évalua que les prêts au public s'élevaient à 3600 par mois en 1917 (30 ooo romans et io 000 " ouvrages sérieux » pour l'année, écrit-il). En réponse à un questionnaire daté du 26 mars 1947, Georges-Émile Marquis précisait que le lectorat s'était élevé à environ 2500 personnes en 1946, dont 1800 étaient de l'extérieur. Pendant le mandat de Jean-Charles Bonenfant (1952-1968), les amateurs de livres d'agrément, comme l'écrit ce dernier, se tournèrent de plus en plus vers la bibliothèque de l'Institut canadien. De fait, les années qui suivirent furent marquées par une spécialisation des collections - politique d'acquisition en amont ; émondage en $\mathrm{aval}^{13}$ - et par une réorientation des lecteurs. Mais la documentation et l'équipement audiovisuel et informatique restent, sur place, à la disposition des citoyens comme le corpus d'informations parlementaires rétrospectives ${ }^{14}$ l'est à distance.

En 2001, l'Assemblée nationale a reconnu officiellement que les richesses de la Bibliothèque sont accessibles aux lecteurs qui en ont besoin et, en mai 2002, le directeur proposa une nouvelle politique de référence et d'accès aux collections, laquelle fut entérinée par les autorités. Cette dernière avait pour but de clarifier le statut de la population en regard de celui de la clientèle prioritaire formée dés élus et de leurs collaborateurs, du lieutenant-gouverneur, des fonctionnaires parlementaires, des journalistes de la Tribune de la presse et les personnes désignées par l'Assemblée nationale : Protecteur du citoyen, Directeur général des élections et Vérificateur général. Ceux-ci ont accès à tous les services de la Bibliothèque.

\section{Chercheurs, professeurs et étudiants}

Une évolution semblable à celle observée du côté du lectorat général fut vécue du côté du va-et-vient

11. Voir le rapport de Alpheus Todd daté du 20 mars 1862

12. Voir le rapport de Pamphile LeMay pour l'année 1889.

13. Particulièrement de la collection romanesque.

14. Biographies des parlementaires, résultats électoraux, composition des ministères, liste des députés par circonscription, lexique parlementaire, bibliographies, dossiers de presse, etc. 
étudiant. Au départ, la Bibliothèque et l'Université Laval furent logées dans le même voisinage. Les conservateurs $y$ enseignaient et certains donnèrent des séances d'initiation à la recherche documentaire à leur bureau. Les conservateurs exigeaient que les étudiants présentent une lettre de leur député et du "supérieur du collège $»^{15}$. Dans les années 1940, ils prêtèrent des lots de volumes à la jeune faculté des Sciences sociales. Puis, l'Université déménagea en banlieue et, simultanément, on assista à la professionnalisation et à l'enrichissement de la bibliothèque universitaire. Dans son rapport annuel, daté du 14 janvier 1959, J.C. Bonenfant note que «le développement considérable de la bibliothèque de l'Université Laval a permis aux professeurs et aux étudiants de trouver à cette bibliothèque ce qu'ils consultaient autrefois à notre bibliothèque ".

A partir de ce moment-là, les chercheurs et tous ceux qui avaient à compulser un corpus documentaire conservé à la B.A.N.Q. prirent le relais des groupes. Les témoignages de certains d'entre eux (André Labarrère, Louis-Edmond Hamelin, André Gaulin, Claude Galarneau, Alain Combes, Aurélien Boivin) furent publiés à l'occasion du Deuxième centenaire de la bibliothèque ${ }^{16}$. Encore aujourd'hui, des chercheurs travaillent sur place pendant des semaines ou des mois au dépouillement d'un journal, des archives personnelles de député, de mémoires présentés aux commissions parlementaires ou à des commissions d'enquête, des discours des hommes et femmes politiques ou, encore, à la lecture d'une édition princeps de la collection Chauveau ${ }^{17}$. Les documents conservés ici, leur organisation, l'indexation qu'on en fait en vue d'en faciliter l'usage aux parlementaires et à leurs collaborateurs facilitent, par le fait même, le travail de nombreux chercheurs.

\section{Conclusion}

La Bibliothèque de l'Assemblée nationale du Québec a maintenant une mission propre et des mandats exclusifs. Des services sont réservés à la clientèle prioritaire ${ }^{18}$. Les collections ont été élaguées et enrichies en fonction de ses besoins. Au cours des ans, on aura fait des efforts pour combler les besoins documentaires d'employés de la Fonction publique ${ }^{19}$ ou du lectorat de la capitale. Petit à petit, le principe de subsidiarité s'est imposé : les ministères, la ville et l'université ont mis des services documentaires adaptés à la portée de leur propre lectorat.

La Bibliothèque de l'Assemblée nationale demeure cependant une bibliothèque de référence et de consultation : les citoyens y sont admis comme les fonctionnaires et les chercheurs. L'accueil réservé à tous est illustré par les nombreuses activités publiques (lancements de livres, expositions, tables rondes, etc.) rendues possibles à la suite du réaménagement de 2001.

Les hommes et les femmes politiques ont défendu cette vision et cette pratique, tant au $19^{\mathrm{e}}$ qu'au $20^{\mathrm{e}}$ siècle, laquelle a été entérinée par les bibliothécaires ${ }^{20}$. Récemment, l'ex-premier ministre Bernard Landry a affirmé, à l'occasion du deuxième centenaire de la B.A.N.Q. : « ... la bibliothèque de la maison du peuple doit être ouverte au public. " Quelques semaines plus tard, le secrétaire général de l'Assemblée nationale édictait : « La Bibliothèque... accueille tous les citoyens du Québec qui effectuent des recherches sur des sujets qui requièrent l'utilisation des documents qu'elle détient. $~^{21}$ En somme, on peut dire que la Bibliothèque s'avère une institution fondamentale dans le processus démocratique, tant celui observé à l'Assemblée que celui vécu dans l'ensemble de la société. $(-)$

\section{Bibliographie}

ACBLF (Association canadienne des bibliothécaires de langue française). 1965. Comité de planification des bibliothèques gouvernementales 1964-1965; section Québec.

ACBLF. 1969. Comité de planification... Rapport final : 10.

Association des secrétaires généraux des parlements. 1974. Les bibliothèques des Parlements. $3^{\mathrm{e}}$ série, $\mathrm{n}^{\circ} 97$ ( $\mathrm{1}^{\mathrm{er}}$ trimestre) : 1-30.

Gallichan, Gilles. 1987. Une institution aux multiples vocations : la Bibliothèque de l'Assemblée nationale, Bulletin de la Bibliothèque de l'Assemblée nationale (du Québec), vol. 16, $\mathrm{n}^{\text {os }}$ 3-4 (janvier 1987) : 5-8.

Pelletier, Jean-Guy. 2002. Témoignages sur la Bibliothèques. Bibliographie et documentation, 45. Québec: Bibliothèque de l'Assemblée nationale, 2002. $106 \mathrm{p}$.

Tanfield, Jennifer. 2000. Parliamentary library, research and information services in Western Europe. Bruxelles : CERDP : 295.

Union interparlementaire. 1987. Les Parlements dans le monde; recueil de données comparatives, $2^{\mathrm{e}}$ éd. Bruxelles : Bruylant : 2 vol.
15. " ... les étudiants... doivent non seulement obtenir la recommandation du député... - puisque c'est là une partie du patronage que celui-ci exerce - mais aussi celle du supérieur du collège » (AAN, Fonds Marquis, dossier 3.5 : lettre datée du 20 septembre 1943 et adressée à Robert Bernier, c.j.m.).

16. Pelletier, Jean-Guy. 2002.

17. Bibliothèque du premier Premier ministre du Québec (1867-1871) et première bibliothèque classée par l'État en 2003.

18. Préparation d'études, de notes d'intervention ou d'information, etc.

19. Voir le témoignage du sénateur Roch Bolduc. Ce dernier remit au conservateur une liste bibliographique d'ouvrages sur l'administration publique à son retour de Chicago en 1953, lequel s'empressa de les acquérir (Pelletier, Jean-Guy. $2002: 29$ ).
20. Témoignage de Pamphile LeMay : «... les bibliothèques manquent. C'est pourquoi, avec l'approbation des honorables présidents des Chambres, j'ouvre la vôtre toute grande aux esprits studieux » (Rapport annuel 1889; Débats du Conseil législatif $1890: 14$ ). On retrouve le même témoignage dans le rapport de 1888.

21. Nouvelles de l'Asted, vol, 21, $\mathrm{n}^{\circ} 4$, juillet-septembre $2002: 11$. Il est vrai que pendant la décennie précédente, on avait hésité à diffuser le catalogue informatisé - alors qu'au XIX'e siècle le catalogue imprimé l'était - et même à considérer les collaborateurs des députés en circonscription comme des abonnés de la Bibliothèque. 